the Witwatersrand, Johannesburg, South Africa; ${ }^{3}$ Division of Medical Microbiology, University of Cape Town, Cape Town, South Africa

Background Acyclovir (ACV) is the first-line treatment for symptomatic primary and recurrent genital herpes in South Africa. Resistance to ACV is mainly due to mutations in the HSV-2 viral UL23 gene that codes for thymidine kinase (TK), resulting in an inability for the drug to inhibit viral replication. The objectives of this study were to obtain genotypic data on the frequency of HSV-2 TK resistance mutations and natural polymorphism as well as to assess the prevalence of ACV-resistant HSV-2 among participants in the genital ulcer aetiological studies conducted between 2007 and 2011.

Methods We amplified and fully sequenced the UL23 gene of 254 HSV-2 positive specimens obtained from participants in genital ulcer aetiological studies conducted between 2007 and 2011 in Gauteng Province, South Africa. Additionally, intratypic differences between the HSV-2 were determined based on the number of reiterated sequences located in the non-coding regions of the US1 and US12 genes of HSV-2.

Results We identified 63 single and/or double nucleotide mutations in the UL23 genes analysed, that resulted in 30 silent mutations and 32 amino acid changes. $41 \%$ of these amino acid changes were due to natural polymorphism. In addition, we identified 19 unknown amino acid changes in 30 samples that have not been described before. All unknown mutations detected were outside the TK resistance "hotspots", which are renowned sites for ACV resistance mutations to occur. No frameshift mutations or mutations causing stop codons were identified in the UL23 genes of the samples analysed.

Conclusions No evidence was found of known ACV resistance mutations in HSV-2 following the addition of ACV as first-line therapy for genital ulceration in South Africa. Genotyping of HSV-2, based on the length of reiterated sequences in the US1 and US12 genes of HSV-2, revealed a high degree of HSV-2 heterogeneity in this population.

\section{P1.015 ON THE MECHANISMS OF DRUG RESISTANCE IN HIV-1 RT}

doi:10.1136/sextrans-2013-051184.0236

B Sharma. University of Allahabaad, Allahabaad, India

Human Immunodeficiency Virus Reverse Transcriptase (HIV-1RT), catalysing synthesis of proviral cDNA has been widely exploited as a most suitable target for attack by anti-AIDS chemotherapeutics. The antiHIV-1RT molecules involve dideoxy analogues of dNTP (nucleoside reverse transcriptase inhibitors, NRTIs), which inhibit it competitively by terminating DNA chain extension. The other group of HIV-1 RT inhibitors involves non-nucleoside reverse transcriptase inhibitors (NNRTIs), which bind preferably in the hydrophobic pocket or elsewhere at enzyme surface, inhibit it non-competitively. The AIDS therapy prescribes different combinations of various anti-HIV drugs mainly the antiHIV-1RTs and antiproteases (in highly active antiretroviral therapy; HAART), which significantly reduces HIV count to undetectable level. Unfortunately, due to drug selection pressure drug resistant variants of HIV-1 emerge and preclude chemotherapy of this pandemic. This paper is an endeavour to illustrate possible mechanisms involved in anti-HIV drugs resistance. Apart from attributes of numerous mutations reported in three key enzymes of HIV (RT, protease and integrase) in making antiHIV drugs, one plausible mechanism of nucleoside analogue resistance involves ATP/GTP-based excision to unblock chain-terminated primers; thereby allowing viral replication to continue. Such unblocking has also been reported in vitro in presence of excess of pyrophosphate concentration; a reaction known as pyrophosphorolysis. In addition to viral mutants isolated from the AIDS patients, we have shown some artificial mutants of K154, which exhibited antiHIV-1 drugs resistance property and the mechanism involved reduction in binding of the drugs to RT because of conformational change. Our recent report on mechanism of resistance of HIV-1RT against rilpivirin due to E138K mutation proposes a new aspect in this context. However, an extensive study exploring some new targets and drug resistance mechanisms is needed for design and development of novel and potential anti-viral agents to combat this challenge

\section{P1.016 MOLECULAR ANALYSIS OF ANTIMICROBIAL RESISTANT NEISSERIA GONORRHOEA ISOLATES FROM DELHI, INDIA: A FUNCTIONAL GENOMICS APPROACH}

doi:10.1136/sextrans-2013-051184.0237

'D Sachdev, ${ }^{2} \mathrm{M}$ Bala, 'I kumari, 'R Pandey, ${ }^{3} \mathbf{U}$ Chaudhry, 'D Saluja. 'Dr. B.R. Ambedkar Center for Biomedical Research, University of Delhi, Delhi-110007, India; ${ }^{2}$ Regional STD Teaching, Training \& Research Centre, Vardhman Mahavir Medical College \& Safdarjung Hospital, NewDelhi-110029, India; ${ }^{3}$ Bhaskarcharya College of Applied Sciences, University of Delhi, NewDelhi-110075, India

Background In India, knowledge regarding N. gonorrhoeae antimicrobial resistance profiling is limited, and data concerning genetic characteristics of N. gonorrhoeae is also lacking. Herein, we investigated the genetic resistance determinants for various antimicrobials used against N. gonorrhoeae isolated in Delhi, India. Various studies have shown that this resistance towards antimicrobials could be either plasmid or chromosomal mediated involving mutations in various genes.

Methods Molecular basis of plasmid and chromosomal mediated antimicrobial resistance was analysed by amplifying and sequencing the most target genes, pen $\mathrm{A}$ and por $\mathrm{B}$, of $\mathrm{N}$. gonorrhoeae. Attempts have been made to in-silico model the structure of mutant PenA to understand how mutations in these genes affect the drug binding. A PCR assay was also carried out to analyse the penicillinase producing $\mathrm{N}$. gonorrhoeae (PPNG).

Results Out of the 40 clinical isolates of N. gonorrhoeae studied which were resistant to various antimicrobials, twenty eight isolates showed high resistance to penicillin $(3-32 \mu \mathrm{g} / \mathrm{ml})$. These resistant isolates were PPNG positive $(70 \% ; 28 / 40)$ and predominantly harboured the African type of PPNG plasmid. Only two isolates carried the Asian type of plasmid. Mutations were also observed in penA and porB genes which correlate their effects on drug resistance. Through in silico modelling studies, we were able to even show that a single point mutation at G452S in penA gene changed the susceptibility of $\mathrm{N}$. gonorrhoeae towards penicillin and tetracycline.

Conclusions This study clearly shows a cumulative effect of increasing mutations with subsequent increase in resistance towards various antimicrobials. Presence of both African and Asian type of penicillinase producing plasmid gives an indication of extensive travel of patients affected with Gonorrhea. Our in silico modelling studies of mutant proteins provide new insights to access increasing antimicrobial resistance among Neisseria gonorrhoeae.

\section{P1.017 UNIQUE ACTIVITY OF SITAFLOXACIN, ONE OF NEWER FLUOROQUINOLONES, AGAINST CIPROFLOXACIN- RESISTANT N. GONORRHOEAE}

doi:10.1136/sextrans-2013-051184.0238

R Hamasuna, T Sho, M Matsumoto, T Matsumoto. Department of Urology, University of Occupational and Environmental Health, Japan, Kitakyushu, Japan

Background Fluoroquinolone-resistance in Neisseria gonorrhoeae is spreading in the world and almost $80 \%$ of $\mathrm{N}$. gonorrhoeae strains are resistant to fluoroquinolones in Japan. It is known that the resistance to fluoroquinolones is closely related to genetic mutations of quinolone-resistance determining regions (ORDR) on gyrase genes such as gyrA or parC. In 2009, the first national surveillance of antimicrobial susceptibilities of $\mathrm{N}$. gonorrhoeae was performed in Japan. In this surveillance, we found that sitafloxacin, one of newer 
fluoroquinolones, had a strong activity to ciprofloxacin-resistant $\mathrm{N}$. gonorrhoeae strains. The MIC90 of ciprofloxacin or sitafloxacin were $16 \mu \mathrm{g} / \mathrm{ml}$ or $0.25 \mu \mathrm{g} / \mathrm{ml}$, respectively.

Purpose In this study, the relationship between genetic mutations of QRDR and antimicrobial susceptibilities of sitafloxacin against ciprofloxacin-resistant $\mathrm{N}$. gonorrhoeae strains was examined.

Methods The subjects were $12 \mathrm{~N}$. gonorrhoeae strains which were gotten by the Japanese national surveillance by three Japanese societies including the Japanese Association of Infectious Diseases, the Japanese Society of Chemotherapy and the Japanese Society of Clinical Microbiology. MICs of sitafloxacin to these 12 strains were more than $2 \mu \mathrm{g} / \mathrm{ml}$, but MICs of sitafloxacin to these strains were less than $0.125 \mu \mathrm{g} / \mathrm{ml}$. The base sequence of $\mathrm{ORDR}$ on gyrA or parC genes of these strains were examined

Results On ORDRof gyrA of 12 strains, mutations of 2 aminoacids were found, such as Ser91 to Phe, Asp95 to Ala or Asp95 to Gly. Regarding parC gene, mutations of 4 amino-acids were found, such as Asp86 to Asn in 1 strain, Ser87 to Asn in 6 strains, Ser87 to Arg in 5 strains, Glu91 to Lys, Gln or Gly in 3 strains and Ala123 to Ser in 3 strains.

Conclusion Sitafloxacin had a strong activity to ciprofloxacinresistant $\mathrm{N}$. gonorrhoeae which had at least more than 3 mutations of amino-acids on $\mathrm{QRDR}$ on gyrA and parC genes.

\section{P1.018 ANALYSIS OF MOSAIC PENICILLIN-BINDING PROTEIN 2 VARIANTS WITH ALA501 MUTATIONS THAT CONFER HIGH-LEVEL RESISTANCE TO EXPANDED-SPECTRUM CEPHALOSPORINS IN NEISSERIA GONORRHOEAE}

doi:10.1136/sextrans-2013-051184.0239

$1 \mathrm{~J}$ Tomberg, ${ }^{2} \mathbf{M}$ Unemo, ${ }^{3} \mathrm{C}$ Davies, ${ }^{1} \mathrm{R}$ Nicholas. ${ }^{1}$ University of North Carolina at Chapel Hill, Chapel Hill, NC, United States; ${ }^{2}$ Orebro University Hospital, Orebro, Sweden; ${ }^{3}$ Medical University of South Carolina, Charleston, SC, United States

Decreased susceptibility to the expanded-spectrum cephalosporins cefixime and ceftriaxone in Neisseria gonorrhoeae has increased dramatically over the past decade globally and recently resistance was reported to the last remaining recommended treatment option, ceftriaxone, raising fears that gonorrhoea may become untreatable. The major reason for decreased susceptibility has been mosaic penA alleles encoding penicillin-binding protein 2 ( $\mathrm{PBP} 2$ ), the major target for these antibiotics, with up to 70 mutations relative to wildtype. Less prevalent in most settings has been non-mosaic penA alleles containing A501V or A501T mutations just downstream of the KTG active site motif that also confer decreased susceptibility. We have shown that Ala501 mutations, when introduced into mosaic penA alleles, confer resistance to expanded-spectrum cephalosporins, and recently, a novel mosaic penA allele containing an A501P mutation and resulting in ceftriaxone resistance was described. To understand the role of Ala501 mutations in mosaic pen $A$ alleles, we transformed FA19 with the mosaic penA allele from $35 / 02$ harbouring a randomised codon at position 501 and selected for increased cefixime resistance. From this screen, we identified five Ala501 mutations (Val, Thr, Ser, Pro and Arg) that resulted in increased cefixime resistance, indicating that only a small subset of mutations are capable of conferring resistance. Surprisingly, only one clone with an A501P mutation was selected, perhaps suggesting a fitness defect with this mutation. MIC analyses showed that mutation of Ala501 to Val, Ser or Thr conferred 2.5-fold increases in resistance, whereas mutation to Arg and Pro increased resistance nearly 5-fold. PBP2-6140CT (PBP2 containing four C-terminal mutations) harbouring A501V or A501T mutations was crystallised and revealed major ordering and some reorganisation of the $\beta 3-\beta 4$ hairpin that is immediately adjacent to the active site. Modeling of $\beta$-lactams into the crystal structure indicates that the mutations likely introduce a steric clash with the R1 substituent of expanded-spectrum cephalosporins.

\section{P1.019 IDENTIFICATION OF THE AMINO ACIDS CONFERRING HIGH-LEVEL RESISTANCE TO EXPANDED-SPECTRUM CEPHALOSPORINS IN THE PENA GENE FROM THE NEISSERIA GONORRHOEAE STRAIN H041}

doi:10.1136/sextrans-2013-051184.0240

1J Tomberg, ${ }^{2} \mathbf{M}$ Unemo, ${ }^{3} \mathrm{M}$ Ohnishi, ${ }^{4} \mathrm{C}$ Davies, 'R Nicholas. 'University of North Carolina at Chapel Hill, Chapel Hill, NC, United States; ${ }^{2}$ Orebro University Hospital, Orebro, Sweden; ${ }^{3}$ National Institute of Infectious Diseases, Tokyo, Japan; ${ }^{4}$ Medical University of South Carolina, Charleston, SC, United States

The recent identification of a high-level ceftriaxone-resistant (MIC $=2-4 \mu \mathrm{g} / \mathrm{ml}$ ) isolate of Neisseria gonorrhoeae from Japan (H041) portends the loss of ceftriaxone as an effective treatment for gonococcal infections. This is of grave concern because ceftriaxone is the last remaining option for first-line empiric antimicrobial monotherapy. The penA gene from H041 (penA41) is a mosaic penA allele similar to mosaic penA alleles conferring intermediate-level cephalosporin resistance $\left(\mathrm{Ceph}^{\mathrm{I}}\right)$ worldwide, but has 13 additional mutations compared to the mosaic penA gene from the previously studied Ceph ${ }^{1}$ strain, 35/02 (penA35). When transformed into the wild-type strain FA19, the penA41 allele confers 300- and 570-fold increases in the MIC of ceftriaxone and cefixime, respectively. In order to understand the mechanisms involved in high-level ceftriaxone resistance and to improve the surveillance and epidemiology during the potential emergence of ceftriaxone resistance, we sought to identify the minimum number of amino acid alterations above those in penA35 that confer high-level resistance to ceftriaxone. Using restriction-fragment exchange and site-directed mutagenesis, we identified three mutations - A311V, T316P, and T483S - that, when incorporated into the mosaic penA 35 allele, confer essentially all of the increased resistance of penA41. Mapping these onto the crystal structure of PBP 2 shows that A311V and T316P are close to the active-site nucleophile, Ser310, that forms the acyl-enzyme complex, while Thr483 lies on a loop close to the active site and is predicted to interact with the carboxylate of the beta-lactam antibiotic. These three mutations have thus far only been described in penA41, but dissemination of these in other mosaic alleles would spell the end of ceftriaxone as an effective treatment for gonococcal infections.

\section{P1.020 PHENOTYPIC AND GENETIC CHARACTERIZATION OF THE FIRST THREE CASES OF EXTENDED-SPECTRUM CEPHALOSPORIN RESISTANT NEISSERIA GONORRHOEAE INFECTION IN SOUTH AFRICA AND ASSOCIATION WITH CEFIXIME TREATMENT FAILURE}

doi:10.1136/sextrans-2013-051184.0241

${ }^{1}$ L Y E Gumede, ${ }^{2} \mathrm{M}$ Unemo, ${ }^{3} \mathrm{C}$ Sriruttan, ${ }^{2} \mathrm{D}$ Golparian, ${ }^{1} \mathrm{E}$ E Müller, ${ }^{4} \mathrm{~K}$ Rebe, ${ }^{5} \mathrm{D}$ Fick, ${ }^{6} \mathrm{~J}$ de Wet, ${ }^{3} \mathrm{~J}$ Coetzee, ${ }^{17,8} \mathrm{D}$ A Lewis. ${ }^{1}$ National I nstitute for Communicable Diseases, Johannesburg, South Africa; ${ }^{2}$ WHO Collaborating Centre for Gonorrhoea and other STIS, Swedish Reference Laboratory for Pathogenic Neisseria, Örebro University Hospital, Örebro, Sweden, Örebro, Sweden; ${ }^{3}$ Department of Clinical Microbiology, Ampath National Laboratory Services, Centurion, South Africa, Centurion, South Africa; ${ }^{4}$ Anova Health Institute, Ivan Toms Centre for Men's Health, Cape Town, South Africa, Cape Town, South Africa; ${ }^{5}$ Meldene Medicross Clinic, Johannesburg, South Africa, Johannesburg, South Africa; ${ }^{6}$ Springs Medicross Clinic, Springs, South Africa, Springs, South Africa; ${ }^{7}$ Department of Internal Medicine, University of the Witwatersrand, Johannesburg, South Africa, Johannesburg, South Africa; ${ }^{8}$ Division of Medical Microbiology, University of Cape Town Medical School, Cape Town, South Africa, Cape Town, South Africa

Objectives To describe the phenotypic and genetic characteristics of the first three cases of extended-spectrum cephalosporin (ESC) resistant Neisseria gonorrhoeae in South Africa which were associated, in one case, with a verified cefixime treatment failure.

Methods Three ESC resistant $N$. gonorrhoeae isolates were cultured from the urethral discharge of three men-who-have-sexwith-men (MSM), two residing in Johannesburg and one in Cape 